OPEN ACCESS

Edited by:

Florian M. Thieringer,

University Hospital Basel, Switzerland

Reviewed by:

Pier Camillo Parodi,

University of Udine, Italy

Jared Shenson,

University of Texas MD Anderson

Cancer Center, United States

*Correspondence: Max Wilkat

Max.Wilkat@med.uni-duesseldorf.de

Specialty section:

This article was submitted to

Surgical Oncology,

a section of the journal

Frontiers in Oncology

Received: 02 June 2021 Accepted: 27 September 2021 Published: 19 October 2021

Citation:

Wilkat M, Kübler N and Rana M (2021)

Advances in the Resection and

Reconstruction of Midfacial Tumors

Through Computer Assisted Surgery.

Front. Oncol. 11:719528.

doi: 10.3389/fonc.2021.719528

\section{Advances in the Resection and Reconstruction of Midfacial Tumors Through Computer Assisted Surgery}

\author{
Max Wilkat ${ }^{*}$, Norbert Kübler and Majeed Rana \\ Department for Oral \& Maxillofacial Surgery, University Hospital Düsseldorf, Düsseldorf, Germany
}

Curatively intended oncologic surgery is based on a residual-free tumor excision. Since decades, the surgeon's goal of R0-resection has led to radical resections in the anatomical region of the midface because of the three-dimensionally complex anatomy where aesthetically and functionally crucial structures are in close relation. In some cases, this implied aggressive overtreatment with loss of the eye globe. In contrast, undertreatment followed by repeated re-resections can also not be an option. Therefore, the evaluation of the true three-dimensional tumor extent and the intraoperative availability of this information seem critical for a precise, yet substancesparing tumor removal. Computer assisted surgery (CAS) can provide the framework in this context. The present study evaluated the beneficial use of CAS in the treatment of midfacial tumors with special regard to tumor resection and reconstruction. Therefore, 60 patients diagnosed with a malignancy of the upper jaw has been treated, 31 with the use of CAS and 29 conventionally. Comparison of the two groups showed a higher rate of residual-free resections in cases of CAS application. Furthermore, we demonstrate the use of navigated specimen taking called tumor mapping. This procedure enables the transparent, yet precise documentation of three-dimensional tumor borders which paves the way to a more feasible interdisciplinary exchange leading e.g. to a much more focused radiation therapy. Moreover, we evaluated the possibilities of primary midface reconstructions seizing CAS, especially in cases of infiltrated orbital floors. These cases needed reduction of intra-orbital volume due to the tissue loss after resection which could be precisely achieved by CAS. These benefits of CAS in midface reconstruction found expression in positive changes in quality of life. The present work was able to demonstrate that the area of oncological surgery of the midface is a prime example of interface optimization based on the sensible use of computer assistance. The fact that the system makes the patient transparent for the surgeon and the procedure controllable facilitates a more precise and safer treatment oriented to a better outcome.

Keywords: midfacial tumors, computer assisted surgery, navigated resection, computer assisted reconstruction, orbital volume measurement 


\section{INTRODUCTION}

Progress in the planning and implementation of computer assisted surgery (CAS) with the aid of 3D images for the area of the jaw and facial tumors has been recorded over the past two decades, particularly in order to optimize the interfacesupporting procedure in tumor surgery $(1,2)$. Especially during staging, computer-assisted planning and multi-planar as well as -modal evaluation and analysis of data (CT, MRT) have become indispensable and represent an essential pillar of tumor surgery and tumor management.

The complex 3D structures of the face and head require a lot of experience and skill on the part of the surgeon. Without computerassisted planning and navigation, therapy is only possible with difficulty (3-5). Tumor resection is often associated with the loss of essential, functional structures of the face (6). To reconstruct the affected areas of the face, patient-specific autogenic or alloplastic solutions are used. Computer-assisted systems are increasingly used today to determine the optimal anatomical position and for navigation (7). These systems make use of the possibility of visualizing tumors and their boundaries in CT data as well as planning the reconstruction on virtual three-dimensional models, which delivers very good results in individual planning. However, the planning effort and the costs of previous systems are still high and additional tools and engineering know-how are required to perform the segmentation in the various data sets (MRT, CT, СBCT) manually and in a complex manner. In addition to multimodal image analysis, modern image analysis platforms allow the automatic segmentation of tumors $(8,9)$, the extension of this segmentation by a defined safety distance, the simulation of the bony reconstruction, the import of preformed three-dimensional reconstructions such as titanium grid structures, as well as the image fusion of the planning with the postoperative result $(1,10)$. It is suitable for preoperative analysis as well as intraoperative, interactive image information exploitation (navigation, intraoperative CT/CBCT) as well as for connection to other interfaces, such as postoperative tumor staging or radiation planning.

In our study we focused on application possibilities of CAS for oncologic surgery of the midface. These anatomically complex tumors require a highly sophisticated treatment planning in terms of resection and reconstruction. This is enabled through the usage of CAS. Moreover, CAS might contribute to a better interdisciplinary networking and an improved quality of life for the patient.

The task of the present work was therefore to evaluate to what extent oncological surgery of the midface can benefit from the use of CAS and in which areas it is superior to conventional therapy.

\section{MATERIALS AND METHODS}

\section{Study Design}

This prospective clinical study was approved by the local ethics committee at the Hannover Medical School, Germany. The patient collective considered in this work was treated within the period from 2011 to 2019 in the Department for oral and maxillofacial surgery at the Hannover Medical School and at the University Hospital Düsseldorf.

31 patients with tumors of the upper jaw were treated through computer-assisted tumor resection including tumor mapping and primary reconstruction. A retrospectively analyzed patient sample of 29 cases which were treated through conventional methods without the use of computer assisted surgery served as a control group. The patients of the control group were selected with regard to tumor sizes and tumor locations that were comparable with the random sample of 31 patients that had been treated using CAS.The two groups were compared with regard to descriptive data, resection results and quality of life.

Due to the tumor extent, 15 of the above mentioned 31 patients had defects involving the orbit which needed primary orbit reconstruction. These 15 cases of primary reconstruction of the midface involving the orbit were evaluated for the pre- and postoperative volume of the orbit.

\section{Conventional Tumor Resection}

For conventional tumor resection the surgeon evaluated the tumor extent on basis of the clinical findings involving inspection and palpation and the contemplation of the preoperative standard imaging procedure. The resection border was chosen to include a safety margin of 1 centimeter. Afterwards, the resection was performed followed by taking of circular resection-bed driven specimen of the mucosal resection margin and the deep resection margin for frozen section control. The mucosal resection margin was removed with a minimum of two specimen, while the deep resection margin was removed with a minimum of 1 specimen. In cases of extraoral skin resection, the skin resection margin was collected with a minimum of 2 specimen. The anatomical orientation of these taken resection margins were marked by indicating sutures whose orientation was written into the accompanying pathology request document. In case of positive frozen sections further resection was performed in the area indicated by the pathologist. The final resection status which also involved the bony margins was described in the definitive pathology report.

\section{Computer Assisted Surgery - Digital Workflow}

The whole workflow of data acquisition, preoperative planning, tumor resection and mapping using intraoperative real-time navigation as well as reconstruction of midfacial defects involving the orbit after ablative surgery using CAD/CAM is demonstrated (see Figure 1).

\section{Data Acquisition and Preoperative Planning}

The process of digital planning was carried out using the planning software iPlan ${ }^{\circledR}$ (version 3.0.5, Brainlab, Feldkirchen, Germany). With regard to intraoperative navigation-supported tumor mapping and tumor resection, imaging was acquired first (see Figure 2). 

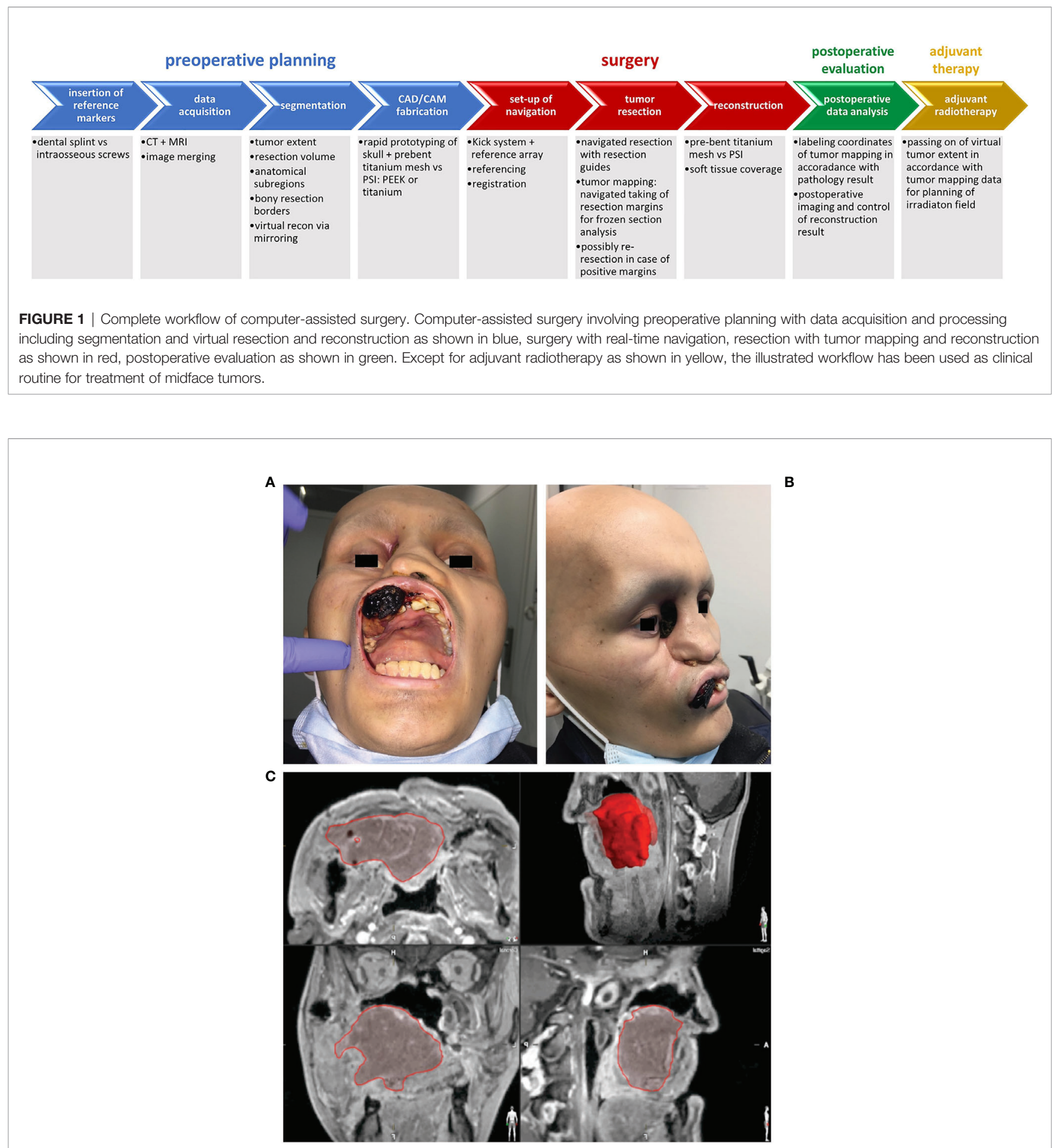

FIGURE 2 | Clinical findings and virtual data in a case of Ewing's sarcoma of the midface. (A) Frontal/intraoral and (B) side view of a 19-year-old patient with an Ewing's sarcoma of the midface presenting with an ulcerating neoplasm in the area of the hard palate, the maxillary vestibule and the right medial nasal slope which has lead to a sinking of the right eyeball. (C) Segmented tumor extent (red) in the multi-planar view of the MRI images.

Imaging data was collected following a standard procedure for all cases. CT data was acquired during arteriovenous phase using contrast medium (field of view $20 \mathrm{~cm}$, pitch 1.0, slice thickness $1.0 \mathrm{~mm}, 140-160 \mathrm{~mA}$, pixel density $512 \times 512$ ). MRI imaging was additionally collected (T1- and T2-weighted images, $1.5 \mathrm{~T}(1 \mathrm{~T}=800 \mathrm{kA} / \mathrm{m})$, slice thickness $2 \mathrm{~mm}$, pixel density $512 \times$ 512) and merged via iPlan ${ }^{\circledR}$ software (version 3.0.5, Brainlab, Feldkirchen, Germany) using the automatic 'best fit' algorithm. 
After automated fusion the result was manually checked in a multi-planar view considering the axial, coronal and sagittal planes. Conformation of best fit was indicated by manually accepting the result.

As soon as the image data were available in the required format, segmentation followed (see Figure 3). Segmentation was performed by the same senior surgeon as investigator for all 31 cases. In particular, the extent of the primary tumor was segmented, and the area of resection was defined by adding a three-dimensional safety margin in a freely selectable distance. Safety margin was set at 1 centimeter. On the basis of the segmented resection volume the bony resection margins were defined and used for designing resection guides. Moreover, anatomical subunits were segmented such as the bony orbit and the alveolar process of the upper jaw which both could be mirrored from the unaffected site for reconstruction purposes if applicable (see Figures 3, 4).

\section{Set Up of Intraoperative Navigation}

During the acquisition of the CT data set, either an individual dental splint with 4 additional titanium mini screws or in cases of a reduced or loosened set of teeth or planned resection which involved more than half of the dental arch in the upper jaw four surgically inserted 2.0 cross-drive titanium mini screws (DePuy Synthes ${ }^{\circledR}$ ) have been used as reference markers during the individual registration of the patient.

Before the operation, the patient was placed in a headrest. The reference star was fixed on the skull via the "Skull ReferenceArray" plus osteosynthesis (see Figure 5A). The reference star was identified by the infrared camera of the navigation system Kick $^{\circledR}$ (Brainlab, Feldkirchen, Germany). identifying the exact position of the patient during operation. As mentioned above the previously inserted reference markers (a dental splint with additional titanium mini screws or titanium mini screws inserted to the skull) were navigated to in order to match their position to the coordinates of the reference markers previously saved in the data record. Additionally, a landmark test was carried out in order to compare the patient on the operating table with the virtual image in the data set and ensure that referencing has been successful.

The time for intraoperative navigation set-up has been measured to evaluate the mean surgery prolongation.

\section{Navigation-Guided Tumor Resection and Tumor Mapping}

The treatment of midfacial tumor patients involving the tumor resection and the reconstruction was performed by only one team with the same senior surgeon being in charge during all CAS operations. Using the navigation probe, the extent of the virtually planned resection could be transferred to the intraoperative situation. Moreover, resection guides indicated the virtually planned resection margins concerning the bony margin (see Figure 6A). After navigated resection of the tumor (see Figures 6B, C), the resection-bed driven margins for frozen section analysis were collected with the aid of navigation by setting intraoperative landmarks with the help of the iPlan software (Version 3.0.5). At least 12 specimens were taken from the mucosal resection margin following the clockwork technique accompanied by at least four specimens from the deep resection margin. In cases of extraoral skin resection further specimen were taken following the clockwork technique. Coordinates of every three-dimensional position of a taken specimen were saved in the software. All these coordinates were thereby integrated into the virtual data to determine the exact three-dimensional position of the taken specimen of the resection margin. Moreover, screenshots of every coordinate
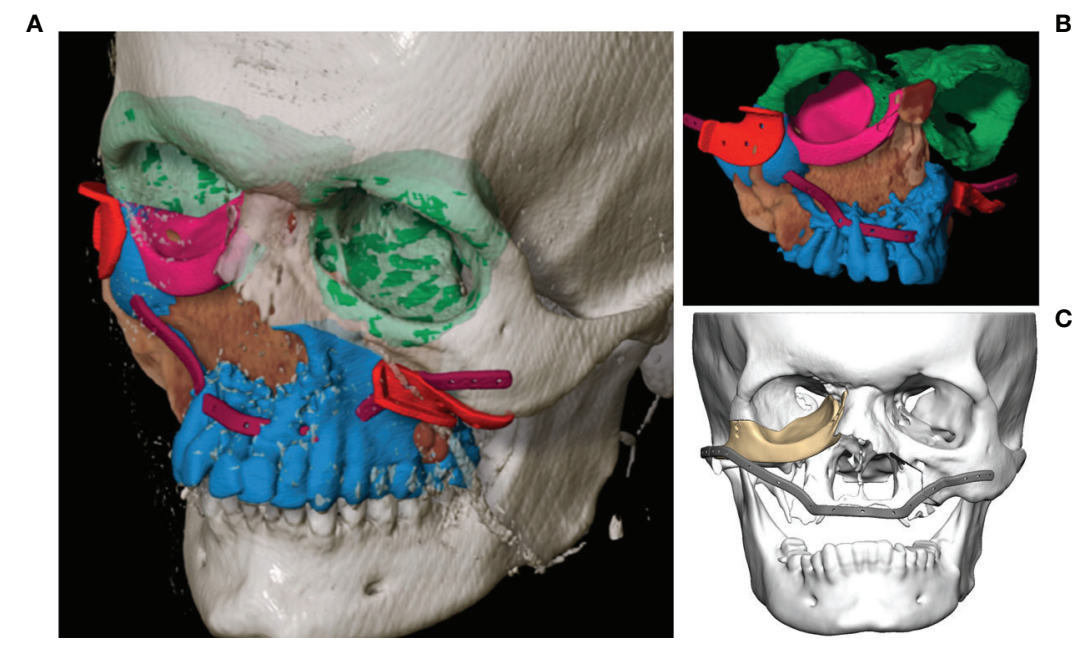

FIGURE 3 | Virtual segmentation and planning of the functional defect reconstruction in a case of Ewing's sarcoma of the midface. (A, B) Segmentation of the tumor extent (brown) and the planned bony resection (blue) with the resection border indicated by the cutting guides (red). The anatomical subunit of the left orbit has been mirrored to reconstruct the right orbit (both green) as a planning template for the right orbital floor reconstruction with a patient specific PEEK implant (pink). The outer contour of the maxilla is reconstructed with a patient specific titanium reconstruction plate (purple). (C) Both PSIs are displayed. 
A

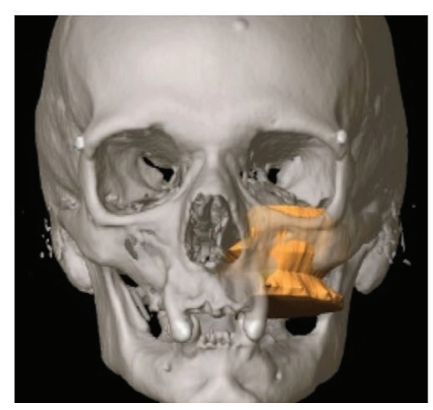

B

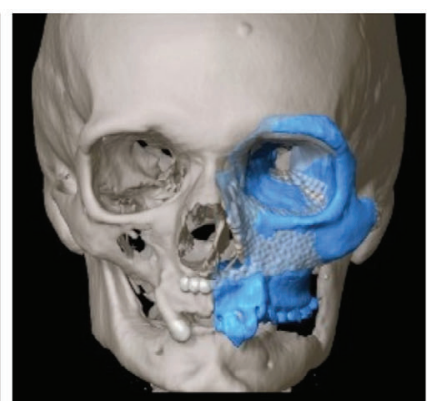

C

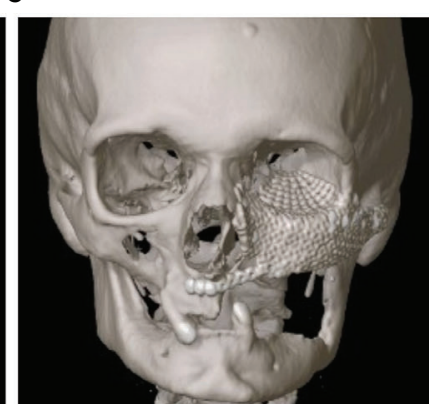

FIGURE 4 | Virtual reconstruction planning using the principle of mirroring. (A) Segmented tumor of the left maxillary sinus with infiltration into the left floor of the orbit (yellow). (B) Template of the healthy right midface mirrored to the left (blue). (C) Postoperative CT shows the reconstruction of the orbit and the midface using two PSIs.

were acquired, printed out and send to the pathologist accompanied by the specimen and the pathology request document to pass on the three-dimensional localization of the taken specimen. The information of specimen position has been linked to the pathology result of the corresponding specimen later on by color-coding: red meaning the specimen was positive for tumor residual, green meaning the specimen was tumor-free (see Figure 5).

In order to evaluate whether the method of intraoperative navigation-assisted tumor resection and mapping was successful in the sense that more complete resections could be carried out, tumor resection of the upper jaw with CAS were compared with the control group. Resection status was defined by the final report of the pathologist. This report considered the main tumor resection specimen - including the bony margins which needed more time for processing compared to soft tissue margins due to the necessary decalcification - as well as the taken resection margin specimen for frozen section analysis. It was differentiated between complete resection (R0), microscopically residual tumor mass (R1) and macroscopically residual tumor mass (R2).

\section{Primary Reconstruction After Ablative Surgery}

The analysis of the tumor and resection margins showed the extent to which the reconstruction was restricted. Midface reconstruction true to original using titanium mesh structures
A

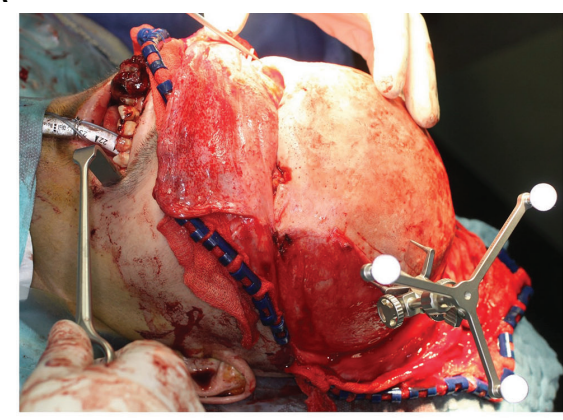

B

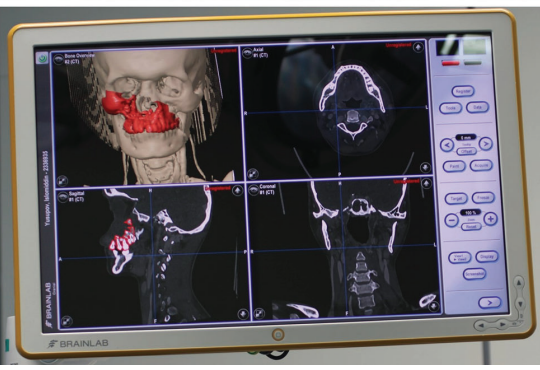

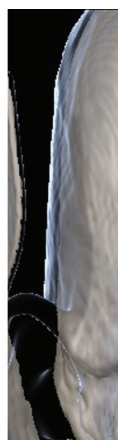

C

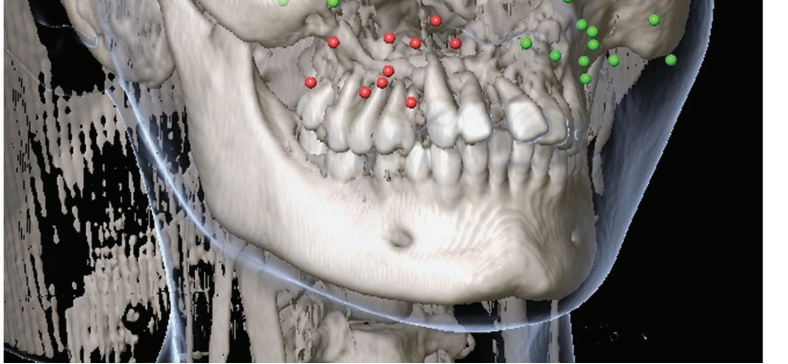

FIGURE 5 | Intraoperative navigation during midfacial tumor ablation in a case of Ewing's sarcoma of the midface. (A) Skull reference array attached for intraoperative navigation after coronal approach. (B) Real time navigation monitor showing the planned bony resection segmented in red color. (C) Data postprocessing of the landmarks set intraoperatively after receipt of the histopathological report (green $=$ tumor-free, red $=$ tumor residual). 


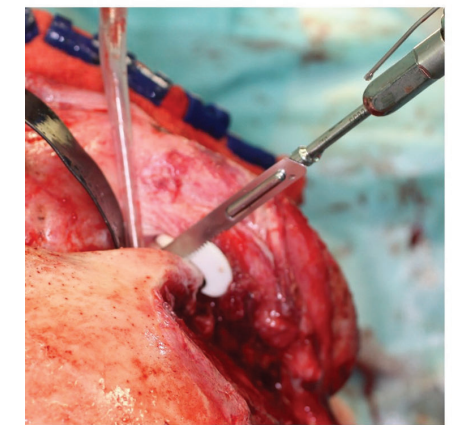

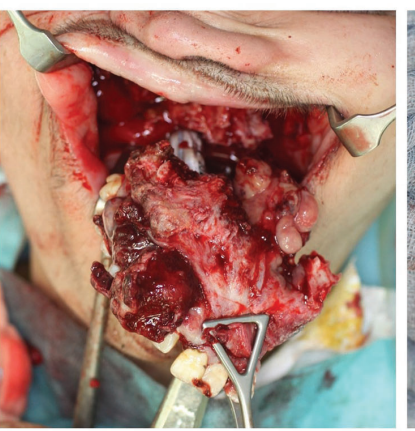

C

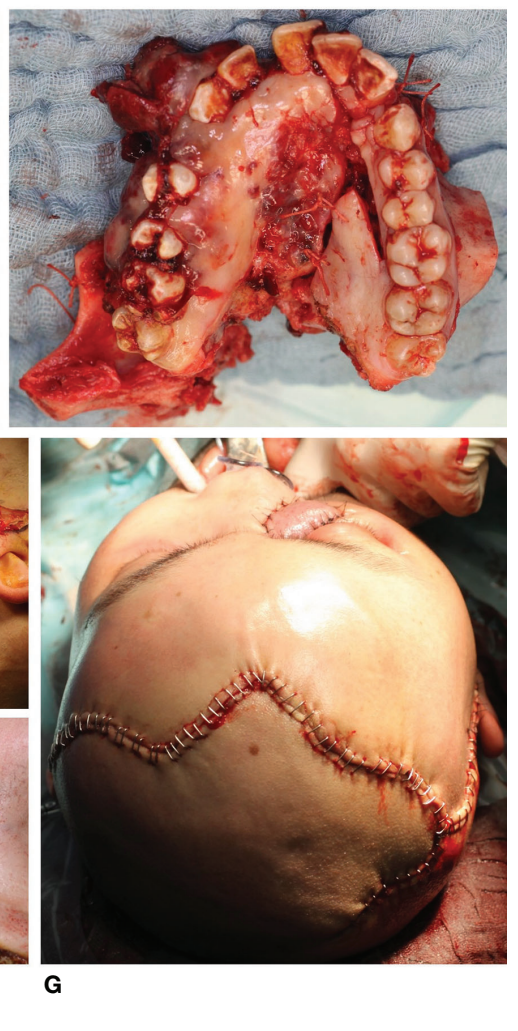

FIGURE 6 | Resection of Ewing's sarcoma of the midface with primarily functional reconstruction. (A) Bone resection using a cutting guide. (B, C) Tumor resection en bloc preserving the eyeball. (D) Primarily functional reconstruction reconstruction of the zygomatic bone and orbit floor using two patient specific implants (combined SLM titanium and PEEK (KLS Martin ${ }^{\circledR}$, Tuttlingen) using the virtually mirrored left orbit as a template) both visible intraorally. (E, F) Dissection of the temporal muscle flap. (G) Wound closure with temporal muscle flap sewn in.

or PEEK implants with soft tissue transplants was the aim (see Figures 6D-G). With unilateral tumor growth the virtual mirroring of the opposite healthy side served as a reference point for the reconstruction of the shape and the volume of the orbit true to the original so that not only the functional but also the aesthetic restoration was achieved (see Figure 4). The virtual reconstruction planning was exported as a stereolithography file (STL format) and printed out three-dimensionally or milled via "rapid prototyping" to create a bio-model made of cheap composite materials, which could also be autoclaved. On the basis of the virtual model a patient-specific 3D implant was preformed by cold forming titanium mesh. Alternatively, a PEEK implant or a titanium implant was manufactured via additive CAD/CAM procedure by KLS Martin (Tuttlingen, Germany) (see Figure 3). During the intraoperative navigation, the reference probe was used to check whether the implant has been inserted anatomically correctly, since the data set acted as a virtual template. For pre-bent titanium meshes the thickness of the mesh itself had to be considered. Therefore an offset according to the used mesh thickness has been used during navigational reconstruction control. In cases of CAD/CAM processed implants the STL-file of the implant itself was integrated into the data set for navigation control.
The software iPlan 4.0 beta was used to measure the intraorbital volume. It is able to virtually subtract a pre-formed cuboid with a defined volume from the segmented bony boundaries so that the volume can be calculated. This algorithm is integrated in the currently available software and enables the surgeon to calculate the volume by simply clicking on the "Orbital cavity" button (see Figure 7).

Patient specific implants were covered by autologous soft tissue transplants (see Figure 8). Transplants were selected according to the resulting soft tissue deficiency to ensure a sufficient soft tissue coverage of the implant to prevent implant exposure.

\section{Determination and Definition of the Irradiation Field}

The use case of integration of the data of tumor mapping into the irradiation protocol has not yet been established as a clinical routine during the time of the treatment of the 31 CAS patients. However, in 3 cases the information of tumor mapping collected intraoperatively was post-processed and forwarded to the radiation therapist. The data was stored in the preoperative data set in XBrain format. After receipt of the histological findings the virtual points were marked in color depending on 

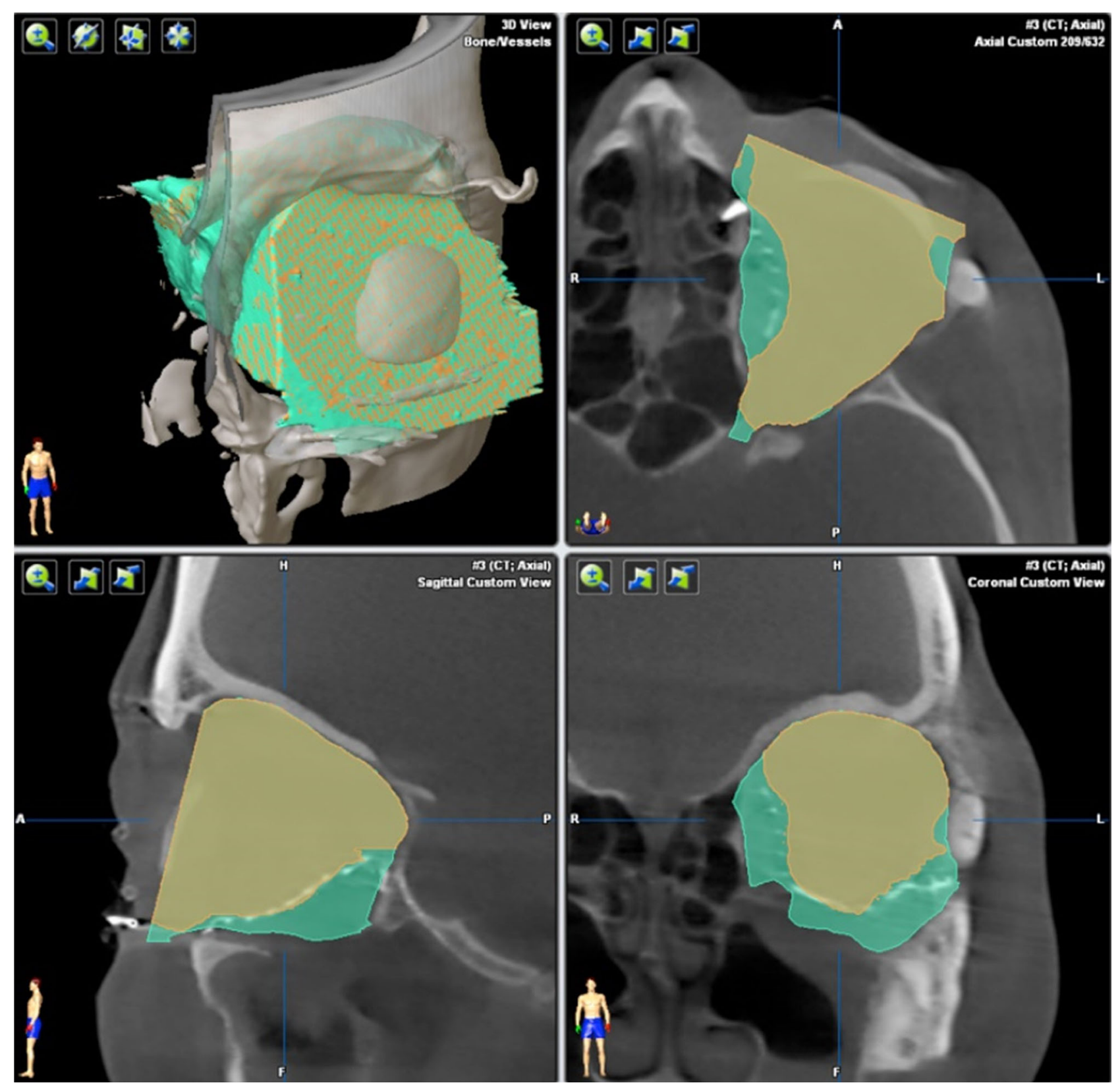

FIGURE 7 | Calculation of orbit volume. Orbital cavity algorithm calculates the orbit volume not only within the bony boundaries (green), but also on the adjacent orbit 3D mesh (yellow). The volume calculation works automatically.

the resection status result. The virtual points that were not within the calculated safety distance were also marked in color accordingly. Thus, the radiation therapist could recognize in the data set which points indicated the supposed residual tumor, which points marked an excessively small safety distance and which points were free of tumor tissue.

After the operative reconstruction of the bony midface, the reconstruction result was checked with the help of a postoperative CBCT by default. The XBrain data was merged with the postoperative CBCT data set. Thus, the preoperative data set, the intraoperative tumor marking, the postoperative data set and the tumor mapping, which had been post-processed depending on the histological findings were available to the radiation therapist as an entire DICOM data set for his irradiation field planning. Moreover, the radiation therapist had the option of merging the data made available by the surgeon with a planning CT for irradiation.

\section{Quality of Life}

The quality of life of patients was measured using the University of Washington Quality of Life Questionnaire (UW-QOL v4) 
A

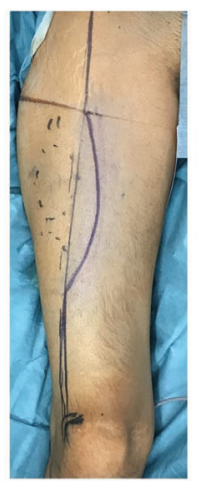

B
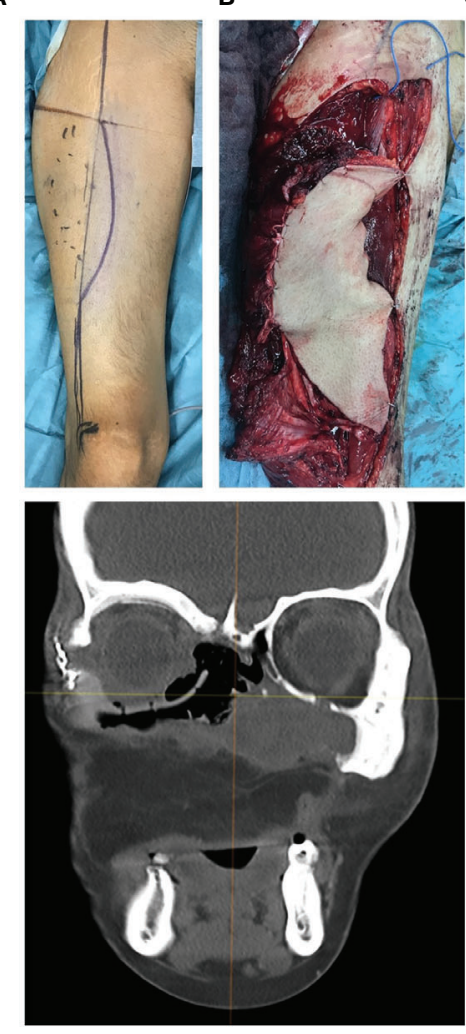

E
C

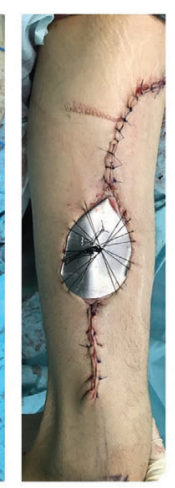

D

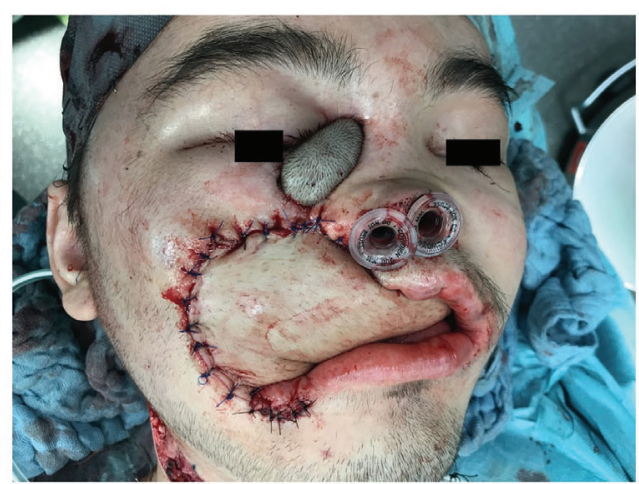

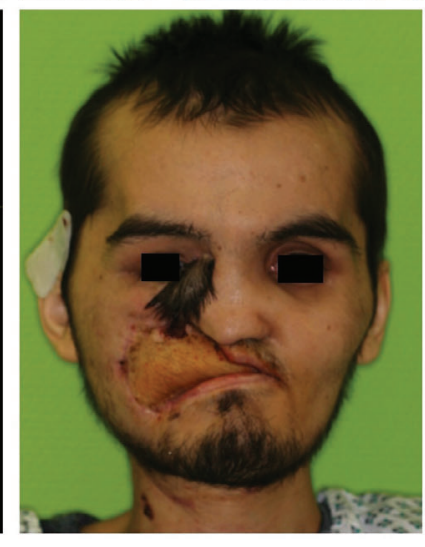

F

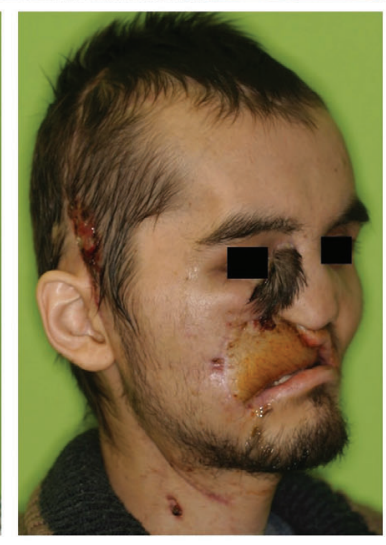

G

FIGURE 8 | Early secondary soft tissue reconstruction of the upper lip and jaw using an anterolateral thigh flap in a case of Ewing's sarcoma of the midface. (A-C) Skin marking, flap harvest and wound closure of the anterolateral thigh flap of the right lower extremity. (D) Anterolateral thigh flap sewed in. (E) Postoperative CT scan in coronal plane shows the patient specific implant in situ reconstructing the right orbital floor while the symmetrical position of the eyeball has been restored. (F) Frontal and (G) half site view of the patient two months after tumor resection and two stage reconstruction (primary orbital floor and early secondary soft tissue of the upper lip and jaw).

(11), a short multifactorial questionnaire that contains specific questions about head and neck tumors. It consists of twelve domains (appearance, activity, recovery, mood, fear, pain, swallowing, chewing, speech, shoulder, taste, saliva). Each of these domains relates to the last seven days and should be rated on a scale that ranges from symptom-free to severe impairment. In addition, the patients should indicate which of these areas was most important to them in the past week. Finally, three global questions were asked about the current state of mind, healthrelated quality of life and overall quality of life, which were to be answered on a five-point Likert scale. The questionnaire has a high level of validity and is particularly suitable for the questions at hand, as it addresses the specific problems of the patient collective $(12,13)$.

\section{Statistical Evaluation}

The statistical analyzes were calculated with SPSS for MAC OS X version 22.0 (SPSS Inc., Chicago, IL, USA). The sociodemographic data were analyzed using descriptive statistics. The mean values and standard deviations are given for intervalscaled data and the frequencies are given for ordinal-scaled data.
In the case of interval-scaled data, t-tests were calculated for independent samples to compare the mean values. The chisquare test was calculated for the ordinal scaled data. A p-value less than 0.05 was considered significant for all tests.

\section{RESULTS}

\section{Time for Set-Up of Intraoperative Navigation}

Table 1 gives an overview of the intraoperative navigation times. The registration process in the form of comparing the instruments with the markers took an average of 3.7 minutes with a standard deviation of 1.1 minutes. The duration of the registration depended on the landmarks used. In 22 cases dental splints were used for referencing. Preoperatively intraosseous inserted, screws were used in 9 cases. There was no significant difference in the duration of the registration process comparing dental splints with intraosseous screws, as the screw heads were not covered by skin after preoperative insertion, which abolished the need for exposure. The registration accuracy was measured in 
TABLE 1 | Time and accuracy of intraoperative navigation.

\begin{tabular}{|c|c|c|}
\hline & $\mathbf{M}(\min )$ & SD (min) \\
\hline \multicolumn{3}{|l|}{ time of } \\
\hline -referencing & 3,2 & 0,3 \\
\hline -registration & 3,7 & 1,1 \\
\hline -intraoperative navigating & 8,8 & 1,3 \\
\hline -export of data & 3,9 & 0,9 \\
\hline \multirow[t]{2}{*}{ total time of intraoperative navigation } & 19,6 & 2,8 \\
\hline & $M(\mathrm{~mm})$ & $\mathrm{SD}(\mathrm{mm})$ \\
\hline accuracy of registration & 1,2 & 0,4 \\
\hline
\end{tabular}

Bold values are the main results.

millimeters and in the case of the present patient collective was $1.2 \mathrm{~mm}$ with a standard deviation of $0.4 \mathrm{~mm}$. The registrations carried out from time to time during the operation to compare the registration accuracy did not show any significant deviations from the original registration accuracy. There was no significant difference in the registration accuracy comparing dental splints with intraosseously inserted screws.

The intra-operative navigation took 8.8 minutes $(\mathrm{SD}=1.3$ min.), The data transfer to the navigation system including the co-registration with the planning data was carried out automatically and took 3.9 minutes ( $\mathrm{SD}=0.9 \mathrm{~min}$.).

While set-up of navigation requires a certain amount of time, navigation itself helps to save time during surgery due to the following aspects: improved orientation in the imaging data set, improved selection of the biopsy position and faster surgery due to the real-time navigation feedback. However, the overall saving of time cannot be quantified due to the lack of a comparison of the highly individual cases. Besides assumably shortened operating time CAS offers also advantages concerning quality control due to instant merging of pre- and intraoperative data sets, high consistency between pre-, intra- and postoperative data sets and automatic data saving for postoperative quality control.

\section{Intraoperative Tumor Marking and Resection}

Table 2 shows the distribution of the descriptive characteristics of the patients with a tumor of the upper jaw treated with CAS as the intervention group compared to those patients treated conventionally without CAS as the control group. The two groups mostly match in their composition. There is no statistically significant difference between the means of the two groups: neither in age calculated by t-test, nor in gender, TNM classification, adjuvant therapy (radiotherapy and chemotherapy), mode of reconstruction or diagnosis checked for by chi-square tests.

In the CAS group, 19 (61.3\%) patients suffered from squamous cell carcinoma, 4 patients (12.9\%) had an adenocystic carcinoma and two patients $(6.6 \%)$ had an ossifying fibroma. One person each (3.2\%) suffered from a keratocyst, a sinunasal carcinoma, a mucoepidemoid carcinoma, a B-cell non-hodgkin lymphoma, an Ewing's sarcoma or an osteosarcoma as displayed in Table 2.

With a number of $16(51.6 \%)$ most patients of the intervention group treated with CAS were reconstructed with a latissimus dorsi flap, three patients $(9.7 \%)$ were reconstructed with a radial forearm flap and for another four patients (12.9\%) a local primary wound closure was sufficient. A serratus anterior muscle flap and an upper arm flap were used in 2 patients $(6.5 \%)$, respectively. One patient $(3.2 \%)$ was reconstructed with a temporal muscle flap, one patient $(3.2 \%)$ with a pedicled palatinal flap and one patient $(3,2 \%)$ received an anterolateral thigh flap. One of the patients (3.2\%) was not reconstructed but received an obturator prosthesis. 8 patients $(25.8 \%)$ received a chemotherapy after the surgical procedure, 23 patients $(74.2 \%)$ a radiotherapy.

In the CAS group, frozen sections samples were collected in higher numbers and smaller sizes compared to the control group. An average of 15.3 frozen sections were collected per CAS patient with an average size of $8.7 \mathrm{~mm}$. In the conventional group an average of 5.3 frozen sections were collected per patient with an average size of $23.2 \mathrm{~mm}$. More R0 resections were achieved in patients treated with CAS in the intervention group compared with the control group as displayed in Figure 9. In the CAS group, an R0 resection could be performed in 22 patients (71.0\%), while in the control group there were 16 patients (55.2\%). An R1 resection was performed in 8 patients $(25.8 \%)$ in the CAS group compared to 12 patients (41.4\%) in the control group. In both groups one patient showed an R2 resection $(\mathrm{CAS}=3.2 \%$; control group $=3.4 \%$ ), which was performed because the tumor had infiltrated the internal carotid artery as a vital structure. However, the differences are not significant $(\mathrm{df}=$ $58 ; \mathrm{t}=-1.112 ; \mathrm{p}=0.271)$.

\section{Reconstruction After Ablative Surgery}

Resection defects after the loss of hard and soft tissue due to ablative surgery of the midface result in considerable functional and aesthetic deficits. These changes are even more dramatic if the orbit is affected by the resection. Once the orbital bony boundaries and/or the intra-orbital soft tissues are impaired, the resulting imbalance between the eyeball and the supporting structures lead to symptoms such as enophthalmus, double vision and facial asymmetry. This particularly applies for orbital prosthesis in cases of anophthalmic patients. Reconstruction of these cases involves a repositioning of the eye globe/prosthesis which might need a reduction of the intra-orbital volume due to intra-orbital soft tissue loss achieved by a reforming of the boundaries of the orbital cavity. Due to the intricate three-dimensional anatomy and the wish for a better prediction of the result, it is advisable to use modern principles of CAS in these cases. CAS allows a backwards planning, which enables the virtual planning of the desired 
TABLE 2 | Test characteristics of patients with tumors of the upper jaw treated with the help of CAS in the intervention group compared to the control group treated conventionally without CAS.

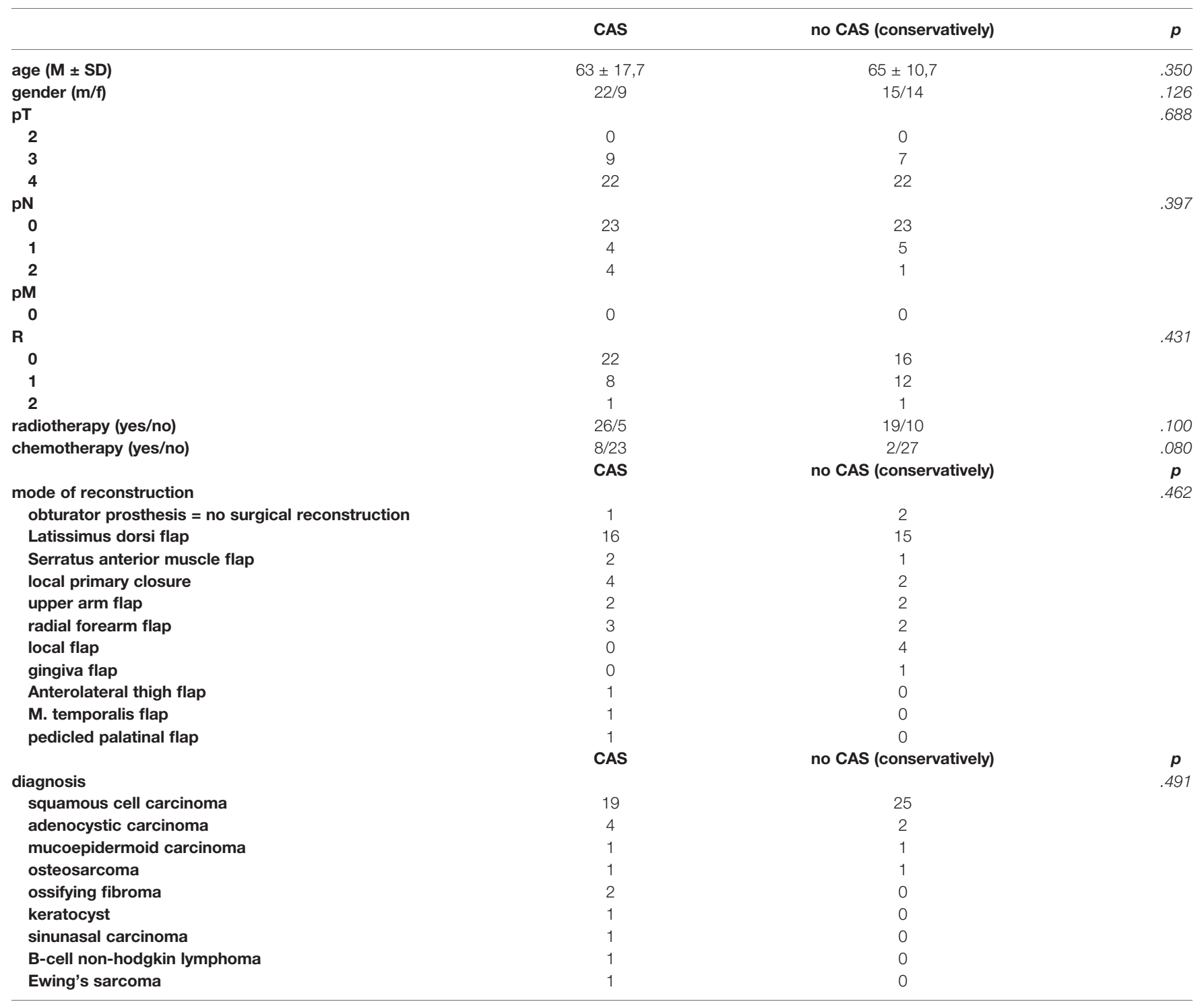

position of the eyeball/eye prosthesis first followed by the calculation of the necessary intra-orbital volume reduction and thus the needed recontouring of the orbital boundaries. This recontouring is achieved via rapid prototyping and prebenting of titanium meshes. The anatomically correct positioning is monitored via intraoperative navigation. The extra-orbital soft tissue defect can be replaced via autologous tissue transfer.

Here we present data of 15 primary cases in need of midfacial reconstruction after ablative tumor surgery involving the orbit. Pre-operative planning took $25 \mathrm{~min}$ for the surgeon and $60 \mathrm{~min}$ for the technical staff on average, while manufacturing of the model and the implant took 2 working days. Table 3 compares the pre- and postoperative volumes of these patients after ablative surgery using CAS. In all cases the intra-orbital volume was reduced, which led to a more symmetrical positioning of and improved support for the eye globe/ prosthesis and thereby for the accompanying structures like the eye lids. The mean preoperative volume for primary reconstructions was $30.97 \mathrm{~cm}^{3}\left(\mathrm{SD}=3.25 \mathrm{~cm}^{3}\right)$ and the postoperative volume was $28.23 \mathrm{~cm}^{3}\left(\mathrm{SD}=3.70 \mathrm{~cm}^{3}\right)$. A t-test for independent samples showed that the mean difference between the pre- and postoperative volumes was significant $(\mathrm{df}=28 ; \mathrm{t}=2.085 ; \mathrm{p}=0.046)$ (see Figure 10).

\section{Optimization of the Interface Between Surgery and Radiation Therapy Using CAPP and CAS}

So far, only the treatment results of three patients could be evaluated, because although the data could be passed on from the surgeon to the radiation therapist, the radiation therapists do 


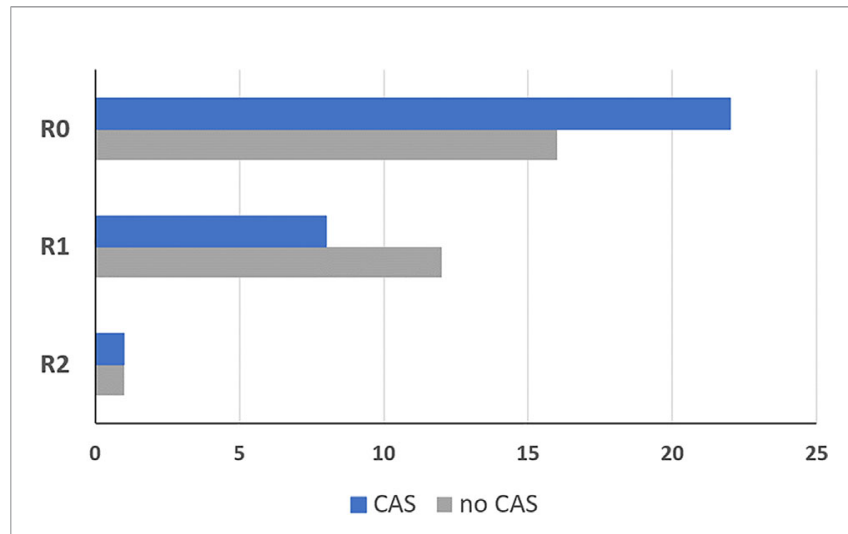

FIGURE 9 | Resection result of the group that was treated using the CAS, compared to the control group. R0: No tumor detectable in the organism R1: Microscopic residual tumor at the resection border R2: Macroscopically tumor or metastases remaining in the patient. Dark blue = intervention group treated with CAS, grey $=$ control group treated without CAS.

not yet routinely include this information, but rather use their planning CTs as a basis for their therapy. However, since the first results are very promising, our efforts are aimed at routinely providing the protocols, which were mainly prepared with the help of the CAS, to the radiotherapists in order to use these protocols as a basis for their therapy. We assume that this procedure will gain a foothold in routine clinical practice. Since this method is still new, the post-processing of the data for passing on to the radiation therapist currently still takes some time. According to previous experiences, this takes about 10 minutes.

\section{Case Study of Selective Irradiation After Virtual Tumor Marking With the Help of CAS}

With the help of this case study, the advantages of intraoperative data collection and transfer to the radiation therapists are

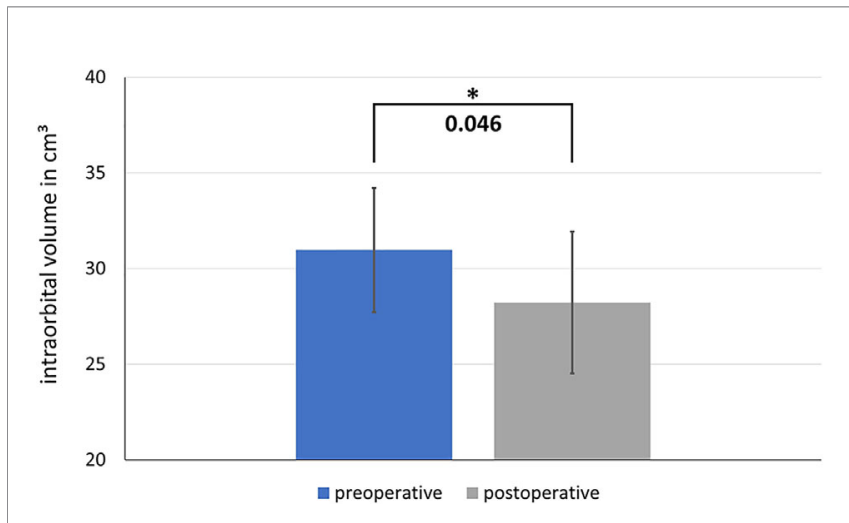

FIGURE 10 | Comparison of the pre- and postoperative intraorbital volumes after ablative surgery and primary reconstruction using CAS. The difference between the preoperative and postoperative volume is significant. * shows significance with a p-value $<0.05$

explained. A 67-year-old patient with a primary SCC of the left maxilla invading the orbital floor (cT4) was operated on using CAS. Intraoperative, navigation-assisted tumor mapping and tumor resection were used. The histopathological findings near the base of the skull still showed parts of a residual tumor in two localizations of the tumor map, there were no close margin areas. This information was highlighted in color in the intra-operative tumor map.

Due to the location of the residual and the reduced general health condition of the patient a further resection could not be performed. Therefore, the files were passed on to the radiation therapist in DICOM format. The iPlan 4.0 beta software was used. Only those coordinates (voxels) that represented a positive histology or a close-margin resection were marked with the Houndsfield unit of $3500 \mathrm{H}$. This value has been chosen well above the usual maximum limit of $3100 \mathrm{H}$ to ensure that the original DICOM data record could not be manipulated any further.

TABLE 3 | Pre- and postoperative volumes of the patients with a primary reconstruction of the orbit using the CAS.

\begin{tabular}{|c|c|c|c|}
\hline patient number & affected site & preoperative volume $\left(\mathrm{cm}^{3}\right)$ & postoperative volume $\left(\mathrm{cm}^{3}\right)$ \\
\hline \multicolumn{4}{|l|}{ primary reconstruction } \\
\hline 1 & $r$ & 30.900 & 23.642 \\
\hline 2 & r & 27.777 & 24.128 \\
\hline 3 & । & 29.826 & 27.174 \\
\hline 4 & I & 32.193 & 30.987 \\
\hline 5 & $r$ & 30.038 & 24.878 \\
\hline 6 & 1 & 28.396 & 25.656 \\
\hline 7 & $r$ & 31.971 & 28.624 \\
\hline 8 & I & 30.378 & 28.375 \\
\hline 9 & । & 39.378 & 36.895 \\
\hline 10 & r & 31.407 & 29.343 \\
\hline 11 & r & 34.535 & 32.535 \\
\hline 12 & r & 25.073 & 23.488 \\
\hline 13 & । & 33.258 & 32.360 \\
\hline 14 & I & 27.517 & 25.912 \\
\hline 15 & I & 31.960 & 29.423 \\
\hline mean & $p=0.046$ & 30.97 & 28.23 \\
\hline standard deviation & & 3.25 & 3.70 \\
\hline
\end{tabular}

Bold values are the main results. 
This provided another advantage as the marked points could be quickly found again using the threshold algorithm. This improved data set was exported as "enhanced DICOM format" (enDICOM) and could be imported into common irradiation devices.

In this case, the enDICOM data set was imported in the Oncentra MasterPlan System (version 3.3, Nucletron, Netherlands/USA) and co-registered with the postoperative radiation therapy planning $\mathrm{CT}$ scan. With this additional information, a more selective irradiation of the midface resulting in smaller volumes of high dose irradiation could be guaranteed.

\section{Quality of Life}

For the calculation, the responses of the intervention group that were treated using CAS for tumors of the upper jaw were compared to the responses from the control group which were treated conventionally without CAS. A mean value comparison was calculated using the t-test for independent samples. Out of the included 60 patients a total of 42 patients fully completed and returned the QoL-questionnaire and thereby were included in the calculation. The group that was treated using CAS had a response rate of $68 \%$ and the control group a response rate of $72 \%$ which results in 21 patients of each group.

Figure 11 shows the distribution of the score rating with regard to the global question about health-related quality of life and overall quality of life. For both, the CAS group tends to have a better scoring (health-related quality of life: $\mathrm{M}=3.70$; $\mathrm{SD}=$ 0.66 , overall quality of life: $M=3.60 ; S D=0.75$ ) than the control group (health-related quality of life: $\mathrm{M}=3.35$; $\mathrm{SD}=0.90$, overall quality of life: $M=3.20$; $S D=1.01$ ). However, the differences do not become significant neither in terms of health-related quality of life ( $\mathrm{df}=38 ; \mathrm{t}=1.872 ; \mathrm{p}=0.069)$ nor for the overall quality of life $(\mathrm{df}=38 ; \mathrm{t}=1.424 ; \mathrm{p}=0.163)$.

When looking at the question of how the patients are doing compared to the months prior to diagnosis, the group treated with CAS $(\mathrm{M}=2.80 ; \mathrm{SD}=1.19)$ tended to have better scores than the control group $(\mathrm{M}=2.65 ; \mathrm{SD}=1.23)$ without the difference becoming significant $(\mathrm{df}=38 ; \mathrm{t}=-0.392 ; \mathrm{p}=0.698)$.

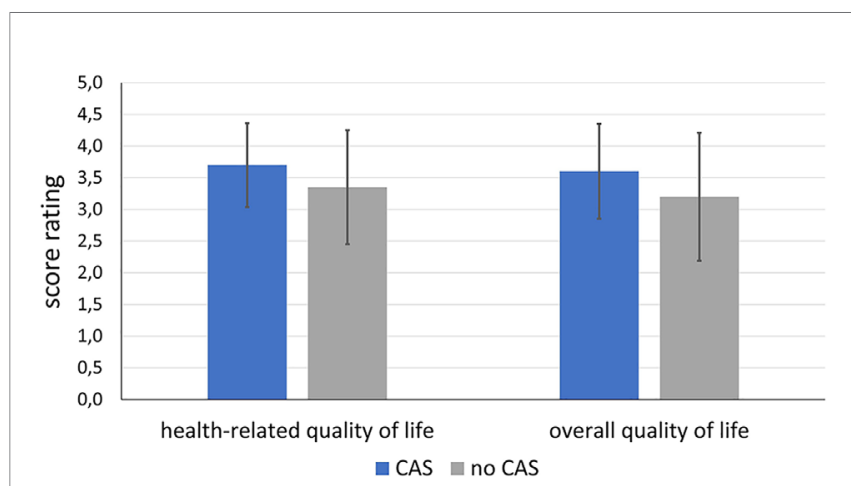

FIGURE 11 | Comparison of health-related and overall quality of life of the patients of the intervention group treated using CAS compared to the control group treated conventionally without CAS.
With regard to the domains, it was found that there tended to be differences in the areas of mood and appearance. In the control group, more patients stated that they were bothered by their appearance and that their mood was impaired. There were no differences between the groups with regard to the other domains. Figure 12 compares the results of both groups with regard to the variables appearance and mood. For the sake of clarity, the items have been combined into two or three statements.

\section{DISCUSSION}

The routine use of CAPP and CAS in the treatment of upper and lower jaw tumors in the maxillofacial surgery was often prevented by the high costs and poor user friendliness due to the high technical complexity and the demanding operation of the systems. Regarding the costs we have the beneficial situation in Germany that the total use of computer-assisted surgery and orbital reconstruction is being reimbursed because of many publications and the clear consensus in the indication having the benefit of precise reconstruction, excellent surgical results and saving time in terms of operation hours.However, there is a need for a comprehensive system with which the entire procedure, from planning to implementation and result control, can be carried out efficiently and easily in order to optimize patient treatment and reduce overall costs. In the present work such a method for the therapy optimization of tumors of the upper jaw and midface area with the necessary treatment steps was presented and evaluated based on the analysis of 31 patients with a tumor of the midface and their surgical treatment with the help of CAS. The preoperative planning, intraoperative navigation with navigated tumor resection and tumor mapping, the reconstruction after ablative surgery and optimization of the interface between surgery and radiation therapy were examined in detail. The CAPP and CAS introduce a new level of quality control and quality assurance and thus promote the professionalization of surgery, because the success of the treatment can now be quantified. From a medicolegal perspective, the surgeon can protect himself externally with the CAPP and CAS, since the documentation of the individual operative sub-steps makes all therapeutic measures transparent, traceable and verifiable at all times. At the same time, the demands on one's own performance increase because the intervention is no longer carried out intuitively or on the basis of experience. Due to the technical possibilities, the aim is to plan and implement the intervention down to the millimeter.

Advantages of these procedures has been presented before (14-18). The current study provides data of a prospective clinical study including 31 patients with midfacial tumors treated with CAS in comparison with 29 patients treated conventionally.

\section{Intraoperative Navigation}

The steps associated with intraoperative navigation such as referencing, registration, intraoperative navigation and data transfer took an average of 19.6 minutes ( $\mathrm{SD}=2.8$ minutes). 

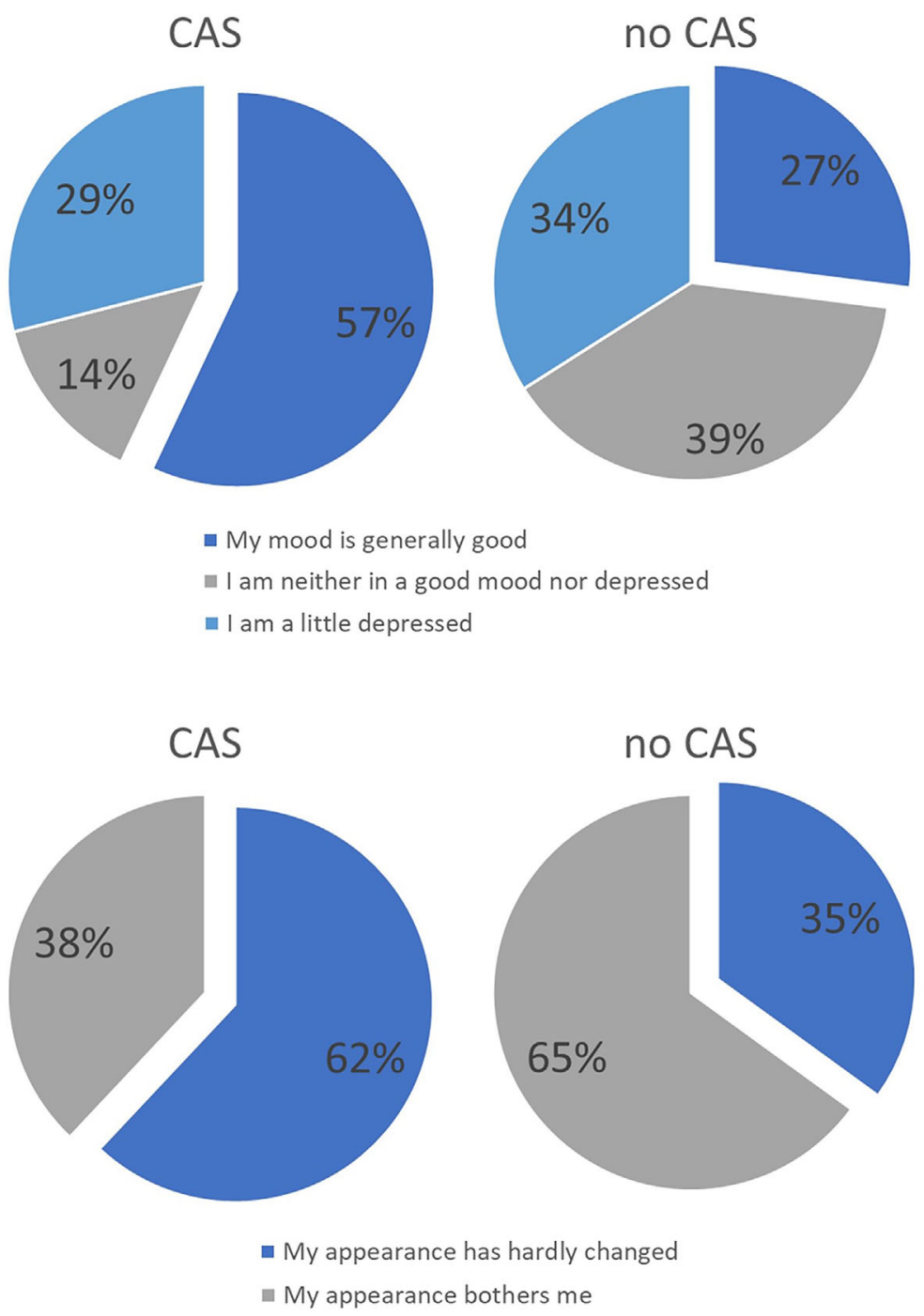

FIGURE 12 | Distribution of answers of the intervention group treated with CAS compared to the control group treated without CAS. In the upper part distribution of answers regarding the question of the mood in recent weeks are displayed while in the lower part distribution of answers regarding the question of 'how you feel about your own external appearance in recent weeks' are shown.

In order to be able to decide whether this additional effort pays off in terms of a cost-benefit ratio, the additional time effort must be compared with indicators of the benefit. Initial studies show that the additional time invested of around 30 minutes predominates $(19,20)$ and that the more experienced the user is with the method of navigation, the quicker the intra-operative set-up of navigation devices (21). First of all, the operating time itself is important, but it is difficult to quantify objectively, as the operating time depends on the patient's individual clinical situation. However, there are already studies that have shown a shortening of the operating time $(22,23)$. In addition to the time saved, the qualitative benefit for the patient should also be taken into account (24). With regard to the time savings, it should be remembered that the detailed preparation for the operation means that all structures are known and that better orientation in the image data set is possible (25). Due to the prior planning and virtual simulation of the access routes, the best access route including alternatives is determined before the operation, so that targeted control of the desired structures is possible, which not only makes the intervention safer, but also reaches the goal faster (26). The reconstruction does not have to be carried out according to the time-consuming "trial and error" procedure in which the best position for the implant has to be selected (27, 28). By planning and adapting the implant beforehand using rapid prototyping, the correct implant position can be controlled directly and immediately adapted using the feedback from the intraoperative navigation (29). It is also positive that the patient is not exposed to further radiation during surgery due to the $\mathrm{x}$ rayless navigation system. A high degree of consistency between the pre-, intra- and postoperative data evaluated through the 
merging of the data sets allows a statement to be made about the success of the intervention and thus makes the procedure more transparent for all involved (30-32). By storing all the data sets, the patient has the benefit of a complete treatment plan, since the radiation therapist can also benefit from the data and can plan his therapy precisely tailored to the patient (33).

\section{Navigation-Assisted Tumor Resection and Tumor Mapping}

A considerable problem of conventional tumor resection without navigation is that the anatomical three-dimensional position of the specimen is described in words written down in the surgery report and in the pathology request document by the operating surgeon. The achieved accuracy can hardly be compared to the method of navigated tumor resection and tumor mapping. This method links the specimen to a coordinate in the three-dimensional anatomy of the patient. Technically this coordinate represents one precise point in space which refers to a sphere-shaped volume with a radius of $1.2 \mathrm{~mm}$ due to the measured registration accuracy during navigation. With a mean specimen size of the taken resection margins of $8.7 \mathrm{~mm}$ the virtual coordinate does not congruently match in full size. However, by taking at least 12 specimen the outline of the resection margin is adequately captured. Together with the histopathological result, this serves as an objective virtual marking of the true tumor extent. This higher number of taken specimen seems only applicable with the help of CAS since differentiation during relocation cannot be achieved with conventional location- and orientation-labeling by simple word description. The orientation-labeling and achieved accuracy of relocation with intraoperative navigation method adequately serves the technical achievable accuracy especially in cases of reresection and should lie above the accuracy of the previous used conventional method. Even in view of soft tissue shifting presenting a well-known problem with intraoperative navigation $(24,34,35)$, the presented method of mapping of midfacial tumors seems still applicable because in the anatomical region of the midface there are many bony structures giving stability to the adjacent soft tissue and thereby limiting the bias of soft tissue shifting.

In combination with the preoperative virtual planning of the resection extent which relies on merging of different imaging modalities combining the different information of these modalities (36), this method of navigated tumor resection and tumor mapping harbors promising results. In our study, we showed that with this method there were more R0 resection possible within tumors of the upper jaw compared to the conventional tumor resection. However, the difference between the two groups was not significant probably due to the sample size. It seems debatable that the observed increase in R0 resections in the CAS group may be achieved by the higher number of frozen sections rather than the use of navigation and CAS. However, in our opinion this high number of frozen sections cannot be adequately labeled with conventional methods allowing precise relocation in case of re-resection. Therefore, a technically applicable sampling of a high number of frozen sections is only reasonably achievable through CAS as already stated above. The comparison of the groups with regard to age, gender, radiation, diagnosis and reconstruction method shows that the groups did not differ in any respect. The CAS is therefore not only indicated for certain patients but can be used in all cases in everyday clinical practice. It has been proven to be particularly advantageous in the case of T4-tumors, since here, a successful operation without the use of CAS in terms of free resection margins is less likely $(37,38)$.

Even though no clear statements can be made about the longterm success of the CAS compared to previous methods, it should be noted that the CAS makes it easier to maintain the safety margin (37-39). Longitudinal studies will show whether the recurrence rate can be reduced in the long term, as this is already the case in other disciplines (40-42).

Another advantage of the CAS is that all information about the patient's anatomy is available preoperatively. This means that it is known how far the safety distance can be maintained before the intervention. If, for example, the safety margin touches the base of the skull, bony structures can be removed or preserved with greater certainty, since the distance to intracranial perforation is known. Our experience has shown that CAS has proven itself particularly well in the midface area, since most tumors are closely related to bony structures. This is precisely why the CAS is particularly suitable for T3 and T4 tumors.

\section{Reconstruction After Ablative Surgery}

The loss of hard and soft tissue after tumor resection is associated with substantial functional and aesthetic deficits (43). CAS in the form of computer-assisted design and models (CAD/CAM) has by far improved the possibilities of reconstructive surgery, especially in the planning of symmetrical aspects of the bony contour $(28,44-47)$. With regard to the primary reconstruction of the midface, the focus of the functional and aesthetic rehabilitation is, in addition to the chewing function, on the reconstruction of the orbital region. Since the secondary reconstruction can be expected to show poorer functional and aesthetic results due to various challenges, the focus should be on the primary reconstruction, in which the implants are inserted directly after the tumor resection. In doing so, not only the resection but also the implant insertion is carried out with the help of a template, since the position of the implant can be planned preoperatively according to the prosthetic situation. With the rapid prototyping or computer-assisted design/ modeling (CAD/CAM) process, it is possible to create a virtual template for unilateral defects using simple processes such as mirroring the unaffected side onto the affected side, through which the midface can be restored (3). If there are bilateral defects, there is also the option of importing the midface from the atlas segmentation and adapting it to the remaining bony structures (elastic and rigid deformation) (48). This is used, for example, for complex tumors of the midface. Once the tumor has been resected, the periorbital region is reconstructed with alloplastic materials and autologous bone grafts. Various CAS procedures can be used here.

These procedures have opened up new possibilities and also a new claim for reconstructive surgery of the facial skull since it is now possible to achieve better aesthetic results through the symmetrical and thus faithful restoration of the removed 
structures. While prior to the introduction of these methods it was primarily a matter of repositioning the vascular or avascular bone in such a way that functionality was restored, the steps necessary to completely restore the face can now be planned preoperatively, taking into account the aesthetic subtleties $(44,45)$.

Our data showed that when the orbit is reconstructed after tumor surgery using CAS, the postoperative volume decreases. The postoperative reduction in volume is needed in cases of intra-orbital soft tissue deficiencies. Moreover, potential soft tissue atrophy and scaring may occur after surgery in this area which can cause enophthalmus. Therefore, we preferred a slight undercorrection rather than an overcorrection But even if the intra-orbital soft tissue was not touched, the postoperative volume was decreased. On the one hand, this is due to the fact, that a new implant is inserted which has its own volume, which must be taken into account. The segmented orbit mesh has an average volume of about $1.3 \mathrm{~cm}^{3}$. If this number was added to the postoperative orbital volume, the difference between the pre- and postoperative volumes would no longer be significant. Deviations are also more likely with regard to the orbit compared to midface reconstruction since the structures are more filigree and complex and manual adjustments are therefore often necessary. On the other hand, the orbit mesh is bent manually using the STL models, so that minor errors can occur.

\section{Successful Irradiation With Computer- Assisted Surgery}

According to Boehm et al. only a few technical solutions for structured data storage and processing of all patient-related data are scientifically described (49). As already described above, after an operation it is necessary to transmit the data to the radiation therapist so that he can optimally plan the therapy. Without the CAS, the radiation therapist has insufficient information available in the form of histological findings, surgical reports and CT data sets, according to which he must plan a strategy. With the help of the CAS, screenshots in form of jpeg files and even enhanced DICOM data sets can be transmitted to the pathologist after the surgical procedure, so that the information can be passed on in a language-independent and unambiguous manner. The 3D volume rendering enables the pathologist to identify the location of the tumor in the image. Based on the histological results, positive boundaries can then be marked in the data set or drawn in in color. As part of further oncological treatment, this data can be saved and transmitted in DICOM format to oncologists, radiologists and pathologists so that they can receive specific tumor information such as the invasion of adjacent structures. Therefore, CAS can serve to enhance the interdisciplinary collaboration and improve patients' outcome. This is especially relevant for the treatment planning of adjuvant radiation therapy. While radiation therapy has been shown to reduce loco-regional recurrence rates (50), it harbors significant side effects such as xerostomia. These side effects occur due to the damaging of healthy tissue surrounding the tumor tissue. The planning of dose distribution during radiation relies on post-operative imaging (51). However, it can be challenging to differentiate on the basis of the imaging data between tissue aberrations due to remaining tumor or due to postoperative changes. The described procedure of tumor mapping can link the pathological information to a definitive three-dimensional coordinate and thereby helps reduce any doubt during interpretation of the imaging data. This allows a more precise dose distribution. However, if this procedure leads to a further reduced loco-regional recurrence rate or to lesser side effects during radiation therapy remains to be seen in further studies focusing on statistically representative patient groups which underwent adjuvant radiation therapy relying on CAS.

\section{Quality of Life}

It is difficult to quantify patients' quality of life in terms of CAS due to various problems. On the one hand, due to the lack of a comparison option, the patient is often not even aware that the CAS, for example, was able to remove the tumor more effectively or that vital structures could be better preserved. In addition due to the fact that patients are suffering from cancer and have to undergo an operation, the level of suffering among patients is so high that the quality of life is impaired for all patients, whether they were operated with or without the use of CAS (52-54). Large numbers of cases would be necessary to calculate significant differences between the groups. However, our data showed that those patients who were operated on using CAS had higher scores, especially in the areas of mood and appearance. These factors in turn correlate with one another.

When asked about the worst event since the operation, patients with oral cancer above all mentioned the realization of facial distortions, even if these seemed minor to third parties $(55,56)$. This shows that the patient's appearance is particularly important, which is understandable in terms of social integration after treatment. Appearance is also related to mood. While in the past the patient's survival was in the foreground and the operation was therefore extremely radical, CAS offers far more options here. By setting a safety distance in advance as part of the preoperative planning, it becomes evident which structures need to be removed, so that the reconstruction of the face can be planned in detail in advance and patient-specific implants can be made. Therefore, there was a tendency towards a higher health-related and overall quality of life, on which the factors appearance and mood exert a decisive influence. In particular for health-related quality of life, our results became almost significant, which suggests that a larger sample might show significant results.

\section{CONCLUSION}

The CAS offers great advantages over the previously established therapy methods in ablative tumor surgery, which are primarily reflected in the precision, safety and success of the treatment. Thanks to sophisticated systems for merging data sets from different imaging processes and the resulting possibilities for precise preoperative planning, in which the treatment goal can be determined in advance, taking into account all the necessary information, the operation can be less invasive while still maintaining a successful and more predictable result. Moreover, the possibility of computer-assisted implementation and 
documentation enables interdisciplinary, language-independent and thus unambiguous communication due to the broad database. Given these advantages of CAS, it is surprising that CAS is not yet routinely used in clinics. Due to the costs and the time required for the implementation and familiarization with the systems it becomes understandable that some surgeons have not yet been convinced of the use of the CAS.

In this study, we showed distinct advantages of CAS in the complex oncologic surgery of the midface. The operation and the planned expected results of the operation can be simulated preoperatively on the computer, afterwards an intraoperative implementation of the simulation is possible with the help of the navigation. The postoperative control through image fusion of the preoperative plan with a postoperative data set enables an evaluation of the reconstruction result with millimeter precision, as it is desired for complex reconstructions.

Another great advantage of this system is that all steps in the diagnosis and therapy of the patient are traceable at any time. If surgical obstacles arise intraoperatively, an alternative procedure with this system can be carried out with a minimum of effort and the complete tumor resection can still be ensured. Thus, the goal of improving the success of the treatment, optimization of interdisciplinary collaboration and the patient's quality of life through complete tumor resection and adequate reconstruction is retained.

\section{REFERENCES}

1. Schramm A, Suarez-Cunqueiro MM, Barth EL, Essig H, Bormann K-H, Kokemueller H, et al. Computer-Assisted Navigation in Craniomaxillofacial Tumors. J Craniofac Surg (2008) 19:1067-74. doi: 10.1097/SCS. $0 \mathrm{~b} 013 \mathrm{e} 3181760 \mathrm{fc} 0$

2. Strauss G, Winkler D, Trantakis C, Schulz T, Meixensberger J. PostProcessing of Radiological Data for Preoperative Planning in Skull Base Surgery. Laryngo- Rhino- Otologie (2004) 83:157-63. doi: 10.1055/s-2004814271

3. Gellrich N-C, Schramm A, Hammer B, Rojas S, Cufi D, Lagrèze W, et al. Computer-Assisted Secondary Reconstruction of Unilateral Posttraumatic Orbital Deformity. Plast Reconstruct Surg (2002) 110:1417-29. doi: 10.1097/ 01.PRS.0000029807.35391.E5

4. Eckardt A, Swennen GRJ. Virtual Planning of Composite Mandibular Reconstruction With Free Fibula Bone Graft. J Craniofac Surg (2005) 16:1137-40. doi: 10.1097/01.scs.0000186306.32042.96

5. Wong ME, Kau CH, Melville JC, Patel T, Spagnoli DB. Bone Reconstruction Planning Using Computer Technology for Surgical Management of Severe Maxillomandibular Atrophy. Oral Maxillofac Surg Clinics North America (2019) 31:457-72. doi: 10.1016/j.coms.2019.03.008

6. Bell RB. Computer Planning and Intraoperative Navigation in CranioMaxillofacial Surgery. Oral Maxillofac Surg Clinics North Am (2010) 22:135-56. doi: 10.1016/j.coms.2009.10.010

7. Rana M, Essig H, Eckardt AM, Tavassol F, Ruecker M, Schramm A, et al. Advances and Innovations in Computer-Assisted Head and Neck Oncologic Surgery. J Craniofac Surg (2012) 23:272-8. doi: 10.1097/scs.0b013e318241bac7

8. Wilkat M, Singh DD, Lutz I, Möllmann H, Gellrich N-C, Rana M. Use and Evaluation of a Computer-Assisted Examination Method for the Diagnosis and Analysis of Medication-Related Osteonecrosis of the Jaw. Craniomaxillofac Trauma Reconstruct (2020) 14:36-42. doi: 10.1177/ 1943387520922767

9. Rana M, Modrow D, Keuchel J, Chui C, Rana M, Wagner M, et al. Development and Evaluation of an Automatic Tumor Segmentation Tool: A Comparison Between Automatic, Semi-Automatic and Manual

\section{DATA AVAILABILITY STATEMENT}

The raw data supporting the conclusions of this article will be made available by the authors, without undue reservation.

\section{ETHICS STATEMENT}

The studies involving human participants were reviewed and approved by Ethics committee of Medical School of Hannover. The patients/participants provided their written informed consent to participate in this study. Written informed consent was obtained from the individual(s) for the publication of any potentially identifiable images or data included in this article.

\section{AUTHOR CONTRIBUTIONS}

MR conceived of the work and participated in its design and coordination. MW and MR made substantial contributions to data acquisition and conception of manuscript. MW drafted and designed the manuscript. MR has been involved in drafting and revising the manuscript. All authors contributed to the article and approved the submitted version.

Segmentation of Mandibular Odontogenic Cysts and Tumors. J CranioMaxillofac Surg (2015) 43:355-9. doi: 10.1016/j.jcms.2014.12.005

10. Gellrich N-C, Schramm A, Böckmann R, Kugler J. Follow-Up in Patients With Oral Cancer. J Oral Maxillofac Surg (2002) 60:380-6; discussion 387-8. doi: 10.1053/joms.2002.31224. discussion 387-8.

11. Rogers SN, Lowe D. The University of Washington Quality of Life Scale. In: VR Preedy, RR Watson, editors. Handbook of Disease Burdens and Quality of Life Measures. New York, NY: Springer New York. (2010) p. 101-28. doi: 10.1007/978-0-387-78665-0_6

12. Chen AM, Daly ME, Farwell DG, Vazquez E, Courquin J, Lau DH, et al. Quality of Life Among Long-Term Survivors of Head and Neck Cancer Treated by Intensity-Modulated Radiotherapy. JAMA Otolaryngol- Head Neck Surg (2014) 140:129-33. doi: 10.1001/jamaoto.2013.5988

13. D'Antonio LL, Zimmerman GJ, Cella DF, Long SA. Quality of Life and Functional Status Measures in Patients With Head and Neck Cancer. Arch Otolaryngol-head Neck Surg (1996) 122:482-7. doi: 10.1001/archotol. 1996.01890170018005

14. Rana M, Chui CHK, Wagner M, Zimmerer R, Rana M, Gellrich N-C. Increasing the Accuracy of Orbital Reconstruction With Selective Laser-Melted PatientSpecific Implants Combined With Intraoperative Navigation. J Oral Maxillofac Surg (2015) 73:1113-8. doi: 10.1016/j.joms.2015.02.014

15. Zimmerer RM, Ellis E, Aniceto GS, Schramm A, Wagner MEH, Grant MP, et al. A Prospective Multicenter Study to Compare the Precision of Posttraumatic Internal Orbital Reconstruction With Standard Preformed and Individualized Orbital Implants. J Cranio-Maxillofac Surg (2016) 44 (9):1485-97. doi: 10.1016/j.jcms.2016.07.014

16. Schramm A, Suarez-Cunqueiro MM, Rücker M, Kokemueller H, Bormann KH, Metzger MC, et al. Computer-Assisted Therapy in Orbital and Mid-Facial Reconstructions. Int J Med Robotics + Comput Assist Surg: MRCAS (2009) 5 (2):111-24. doi: 10.1002/rcs.245

17. Metzger MC, Hohlweg-Majert B, Schön R, Teschner M, Gellrich N-C, Schmelzeisen R, et al. Verification of Clinical Precision After ComputerAided Reconstruction in Craniomaxillofacial Surgery. Oral Surg Oral Med Oral Pathol Oral Radiol Endodontics (2007) 104(4):e1-10. doi: 10.1016/ j.tripleo.2007.04.015 
18. Essig H, Rana M, Meyer A, Eckardt AM, Kokemueller H, von See C, et al. Virtual 3D Tumor Marking-Exact Intraoperative Coordinate Mapping Improve Post-Operative Radiotherapy. Radiat Oncol (London England) (2011) 6:159. doi: 10.1186/1748-717X-6-159

19. Aponte-Tinao L, Ritacco LE, Ayerza MA, Luis Muscolo D, Albergo JI, Farfall GL. Does Intraoperative Navigation Assistance Improve Bone Tumor Resection and Allograft Reconstruction Results? Clin Orthopaed Related Res (2015) 473:796-804. doi: 10.1007/s11999-014-3604-z

20. Barsa P, Fröhlich R, Sebek F, Suchomel P. Intraoperative CT-Based Spinal Navigation: The Initial 295 Implants Experience. Rozhledy v Chirurgii (2014) 93:16-20. doi: 10.1007/s00701-014-2184-8

21. Farfalli GL, Albergo JI, Ritacco LE, Ayerza MA, Milano FE, Aponte-Tinao LA. What Is the Expected Learning Curve in Computer-Assisted Navigation for Bone Tumor Resection? Clin Orthopaed Related Res (2017) 475:668-75. doi: 10.1007/s11999-016-4761-Z

22. Tan X, Hu M, Tao Y, Luo J, Xu J, Liu S. Application of Computer-Aided Surgery in Fibrous Dysplasia of Cranio-Maxillofacial Bone. Shanghai Kou Qiang Yi Xue = Shanghai J Stomatol (2014) 23:62-5.

23. Sießegger M, Mischkowski RA, Schneider BT, Krug B, Klesper B, Zöller JE. Image Guided Surgical Navigation for Removal of Foreign Bodies in the Head and Neck. J Cranio-Maxillofac Surg (2001) 29:321-5. doi: 10.1054/jcms.2001.0254

24. Anand M, Panwar S. Role of Navigation in Oral and Maxillofacial Surgery: A Surgeon's Perspectives. Clin Cosmetic Investigational Dentist (2021) 13:12739. doi: 10.2147/CCIDE.S299249

25. Choudhri O, Mindea SA, Feroze A, Soudry E, Chang SD, Nayak JV. Experience With Intraoperative Navigation and Imaging During Endoscopic Transnasal Spinal Approaches to the Foramen Magnum and Odontoid. Neurosurg Focus (2014) 36(3):E4. doi: 10.3171/2014.1.FOCUS13533

26. Marmulla R, Niederdellmann H. Precision Control of the Position of the Bone Segments During Surgical Navigation. Rev Stomatol Chirurgie Maxillo-faciale (1998) 99:184-7. doi: 10.1016/S1010-5182(98)80067-X

27. Zhang L, Sun H, Yu H, Yuan H, Shen G, Wang X. Computer-Assisted Orthognathic Surgery Combined With Fibular Osteomyocutaneous Flap Reconstruction to Correct Facial Asymmetry and Maxillary Defects Secondary to Maxillectomy in Childhood. J Craniofac Surg (2013) 24:886-9. doi: 10.1097/SCS.0b013e31827ff370

28. Zhang WB, Wang Y, Liu XJ, Mao C, Guo CB, Yu GY, et al. Reconstruction of Maxillary Defects With Free Fibula Flap Assisted by Computer Techniques. J Cranio-Maxillo-Facial Surg (2015) 43:630-6. doi: 10.1016/j.jcms.2015.03.007

29. Levine JP, Patel A, Saadeh PB, Hirsch DL. Computer-Aided Design and Manufacturing in Craniomaxillofacial Surgery: The New State of the Art. J Craniofac Surg (2012) 23:288-93. doi: 10.1097/SCS.0b013e318241ba92

30. Guevara-Rojas G, Figl M, Schicho K, Seemann R, Traxler H, Vacariu A, et al. Patient-Specific Polyetheretherketone Facial Implants in a Computer-Aided Planning Workflow. J Oral Maxillofac Surg (2014) 72:1801-12. doi: 10.1016/ j.joms.2014.02.013

31. Sun Y, Luebbers HT, Agbaje JO, Schepers S, Vrielinck L, Lambrichts I, et al. Accuracy of Upper Jaw Positioning With Intermediate Splint Fabrication After Virtual Planning in Bimaxillary Orthognathic Surgery. J Craniofac Surg (2013) 24:1871-6. doi: 10.1097/SCS.0b013e31829a80d9

32. Ritacco LE, Milano FE, Farfalli GL, Ayerza MA, Muscolo DL, Aponte-Tinao LA. Accuracy of 3-D Planning and Navigation in Bone Tumor Resection. Orthopedics (2013) 36(7):e942-50. doi: 10.3928/01477447-20130624-27

33. Guijarro-Martínez R, Gellrich NC, Witte J, Tapioles D, Von Briel C, Kolotas C, et al. Optimization of the Interface Between Radiology, Surgery, Radiotherapy, and Pathology in Head and Neck Tumor Surgery: A Navigation-Assisted Multidisciplinary Network. Int J Oral Maxillofac Surg (2014) 43:156-62. doi: 10.1016/j.ijom.2013.09.005

34. Sukegawa S, Kanno T, Furuki Y. Application of Computer-Assisted Navigation Systems in Oral and Maxillofacial Surgery. Jpn Dental Sci Rev (2018) 54:139-49. doi: 10.1016/j.jdsr.2018.03.005

35. Lan L, He Y, An J, Zhang Y. Application of Computer-Aided Navigation Technology in the Extraction of Foreign Body From the Face. J Craniofac Surg (2020) 31:e166-9. doi: 10.1097/SCS.0000000000006126

36. Sun H, Li B, Shen S, Yang T, Zhang L, Shen SGF, et al. Image-Guided Endoscopic Navigation for the Precise Resection of a Mandibular Condylar Osteochondroma. J Craniofac Surg (2013) 24(6):e573-9. doi: 10.1097/ SCS.0b013e31829ad374
37. Ricotta F, Cercenelli L, Battaglia S, Bortolani B, Savastio G, Marcelli E, et al. Navigation-Guided Resection of Maxillary Tumors: Can a New Volumetric Virtual Planning Method Improve Outcomes in Terms of Control of Resection Margins? J Cranio-Maxillofac Surg (2018) 46:2240-7. doi: 10.1016/ j.jcms.2018.09.034

38. Tarsitano A, Ricotta F, Baldino G, Badiali G, Pizzigallo A, Ramieri V, et al. Navigation-Guided Resection of Maxillary Tumours: The Accuracy of ComputerAssisted Surgery in Terms of Control of Resection Margins - A Feasibility Study. J Cranio-Maxillofac Surg (2017) 45:2109-14. doi: 10.1016/j.jcms.2017.09.023

39. Sternheim A, Rotman D, Nayak P, Arkhangorodsky M, Daly MJ, Irish JC, et al. Computer-Assisted Surgical Planning of Complex Bone Tumor Resections Improves Negative Margin Outcomes in a Sawbones Model. Int J Comput Assist Radiol Surg (2021) 16:695-701. doi: 10.1007/s11548-021-02337-w

40. Li F-Y, Chen X-L, Sai X-Y, Zhang J-S, Hu S, Li J-J, et al. Application of Intraoperative Magnetic Resonance Imaging and Multimodal Navigation in Surgical Resection of Glioblastoma. Zhonghua Wai Ke Za Zhi [Chinese J Surgery] (2013) 51(6):542-6.

41. Nandra R, Matharu G, Stevenson J, Parry M, Grimer R, Jeys L. Long-Term Outcomes After an Initial Experience of Computer-Navigated Resection of Primary Pelvic and Sacral Bone Tumours: Soft-Tissue Margins Must be Adequate to Reduce Local Recurrences. Bone Joint J (2019) 101-B:484-90. doi: 10.1302/0301-620X.101B4.BJJ-2018-0981.R1

42. Laitinen MK, Parry MC, Albergo JI, Grimer RJ, Jeys LM. Is Computer Navigation When Used in the Surgery of Iliosacral Pelvic Bone Tumours Safer for the Patient? Bone Joint J (2017) 99-B:261-6. doi: 10.1302/0301620X.99B2.BJJ-2016-0149.R2

43. Kokemueller H, Tavassol F, Ruecker M, Gellrich NC. Complex Midfacial Reconstruction: A Combined Technique of Computer-Assisted Surgery and Microvascular Tissue Transfer. J Oral Maxillofac Surg (2008) 66:2398-406. doi: 10.1016/j.joms.2007.12.030

44. Terzic A, Scolozzi P. Image Guided Surgical Navigation Integrating "Mirroring" Computational Planning Based on Intra-Operative Cone-Beam CT Imaging: A Promising New Approach for Management of Primary Bilateral Midfacial Fractures. Comput Aided Surg (2011) 16:170-80. doi: 10.3109/10929088.2011.581901

45. Hanasono MM, Jacob RF, Bidaut L, Robb GL, Skoracki RJ. Midfacial Reconstruction Using Virtual Planning, Rapid Prototype Modeling, and Stereotactic Navigation. Plast Reconstruct Surg (2010) 126:2002-6. doi: 10.1097/PRS.0b013e3181f447e1

46. Palines PA, Ferrer JR, Yoo A, St Hilaire H, Stalder MW. Simplifying Bony Midface Reconstruction With Patient-Specific Titanium Plates. Plast Reconstruct Surg Global Open (2021) 9:e3555. doi: 10.1097/GOX.0000000000003555

47. Schramm A, Wilde F. Die Computergestützte Gesichtsschädelrekonstruktion. HNO (2011) 59:800-6. doi: 10.1007/s00106-011-2351-7

48. Essig H, Dressel L, Rana M, Rana M, Kokemueller H, Ruecker M, et al. Precision of Posttraumatic Primary Orbital Reconstruction Using Individually Bent Titanium Mesh With and Without Navigation: A Retrospective Study. Head Face Med (2013) 9:1-7. doi: 10.1186/1746-160X-9-18

49. Boehm A, Müller S, Pankau T, Straub G, Bohn S, Fuchs M, et al. Computer Assistance to Improve Therapy Planning for Head Neck Oncology. LaryngoRhino- Otologie (2011) 90:732-8. doi: 10.1055/s-0031-1295410

50. Schiff BA, Roberts DB, El-Naggar A, Garden AS, Myers JN. Selective vs Modified Radical Neck Dissection and Postoperative Radiotherapy vs Observation in the Treatment of Squamous Cell Carcinoma of the Oral Tongue. Arch Otolaryngol-Head Neck Surg (2005) 131(10):874-8. doi: 10.1001/archotol.131.10.874

51. Kessel IL, Blanco A. Update in Radiation Therapy for Oral and Maxillofacial Tumors and Dose Mapping. Oral Maxillofac Surg Clinics North Am (2014) 26 (2):223-9. doi: 10.1016/j.coms.2014.01.008

52. Rhoten BA, Murphy BA, Dietrich MS, Ridner SH. Depressive Symptoms, Social Anxiety, and Perceived Neck Function in Patients With Head and Neck Cancer. Head Neck (2018) 40:1443-52. doi: 10.1002/hed.25129

53. Pandey M, Devi N, Ramdas K, Krishnan R, Kumar V. Higher Distress Relates to Poor Quality of Life in Patients With Head and Neck Cancer. Int J Oral Maxillofac Surg (2009) 38:955-9. doi: 10.1016/j.ijom.2009.04.004

54. Rabbels J, Wyzisk M, Siessegger M, Klesper B, Reuther T, Kübler AC. Quality of Life of Patients With Squamous Cell Carcinoma of the Oral Cavity. Mund-Kieferund Gesichtschirurgie: MKG (2005) 9:300-5. doi: 10.1007/s10006-005-0628-2 
55. Chaturvedi SK, Shenoy A, Prasad KMR, Senthilnathan SM, Premlatha BS. Concerns, Coping and Quality of Life in Head and Neck Cancer Patients. Support Care Cancer (1996) 4:186-90. doi: 10.1007/BF01682338

56. Duffy SA, Ronis DL, Valenstein M, Fowler KE, Lambert MT, Bishop C, et al. Depressive Symptoms, Smoking, Drinking, and Quality of Life Among Head and Neck Cancer Patients. Psychosomatics (2007) 48:142-8. doi: 10.1176/ appi.psy.48.2.142

Conflict of Interest: The authors declare that the research was conducted in the absence of any commercial or financial relationships that could be construed as a potential conflict of interest.
Publisher's Note: All claims expressed in this article are solely those of the authors and do not necessarily represent those of their affiliated organizations, or those of the publisher, the editors and the reviewers. Any product that may be evaluated in this article, or claim that may be made by its manufacturer, is not guaranteed or endorsed by the publisher.

Copyright (c) 2021 Wilkat, Kübler and Rana. This is an open-access article distributed under the terms of the Creative Commons Attribution License (CC BY). The use, distribution or reproduction in other forums is permitted, provided the original author(s) and the copyright owner(s) are credited and that the original publication in this journal is cited, in accordance with accepted academic practice. No use, distribution or reproduction is permitted which does not comply with these terms. 